\title{
Antibiogram of multidrug resistant Acinetobacter baumannii isolated from clinical specimens at King Hussein Medical Centre, Jordan: a retrospective analysis
}

A. Batarseh, ${ }^{1}$ A. Al-Sarhan, ${ }^{7}$ M. Maayteh, ${ }^{2}$ S. Al-Khatirei ${ }^{3}$ and M. Alarmouti ${ }^{3}$

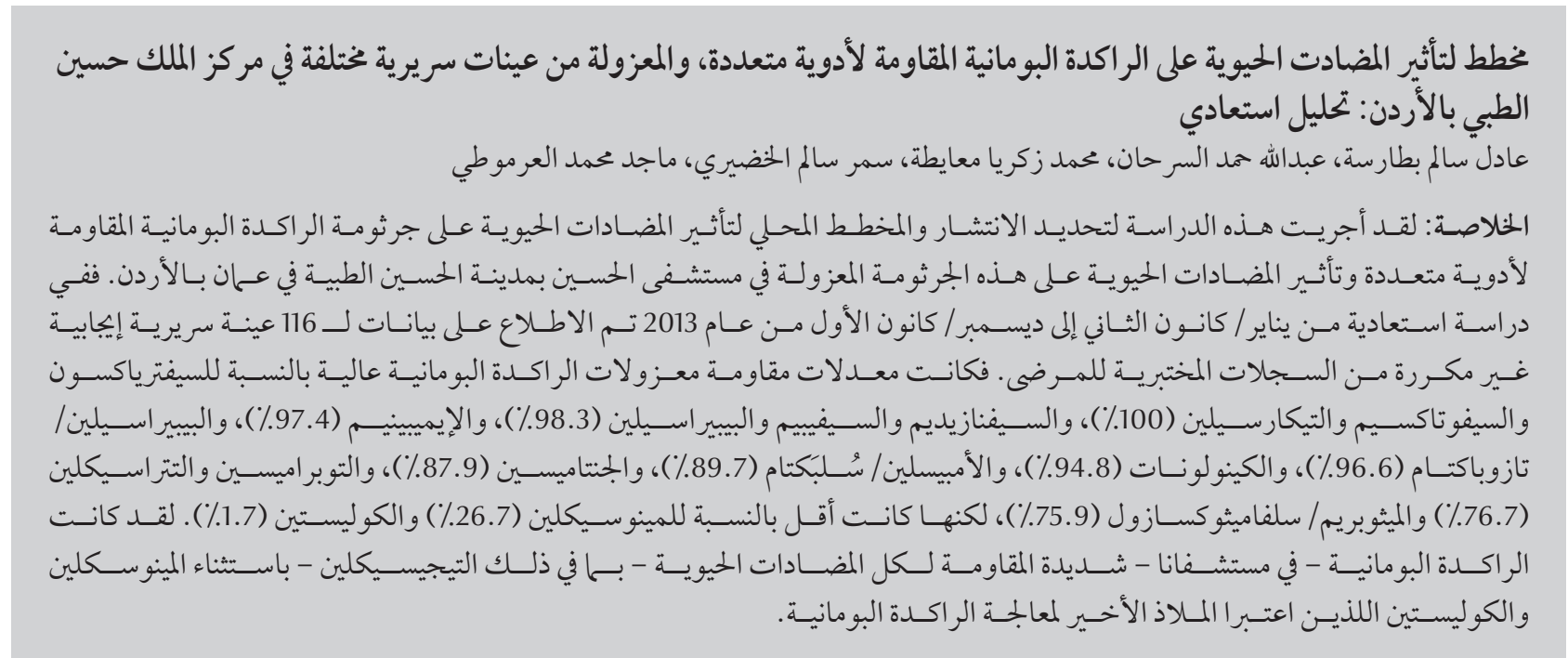

ABSTRACT This study was conducted to determine the prevalence and the local antibiogram of multidrug-resistant Acinetobacter baumannii isolates in Al-Hussein Hospital at King Hussein Medical Centre in Amman, Jordan. In a retrospective study from January to December 2013, data on 116 non-repetitive positive clinical samples were retrieved from patients' laboratory records. The resistance rates of $A$. baumannii isolates were high for ceftriaxone, cefotaxime and ticarcillin (100\%), ceftazidime, cefepime and piperacillin (98.3\%), imipenem (97.4\%), piperacillin/ tazobactam (96.6\%), quinolones (94.8\%), ampicillin/sulbactam (89.7\%), gentamicin, (87.9\%), tobramycin and tetracycline $(76.7 \%)$ and trimethoprim/sulfamethoxazole $(75.9 \%)$, but lower for minocycline (26.7\%) and colistin (1.7\%). A. baumannii in our hospital were highly resistant to all antibiotics, including tigecycline, except for minocycline and colistin which are considered the last resort treatment for multidrug-resistant $A$. baumannii.

Antibiogramme d'isolats d'Acinetobacter baumannii multirésistants à partir de différents échantillons cliniques au Centre médical Roi Hussein en Jordanie : une analyse rétrospective

RÉSUMÉ La présente étude a été menée pour déterminer la prévalence et l'antibiogramme local des isolats d'Acinetobacter baumannii multirésistants à l'hôpital Al-Hussein du Centre médical Roi Hussein à Amman (Jordanie). Dans une étude rétrospective menée de janvier à décembre 2013, les données de 116 échantillons cliniques positifs uniques ont été recueillies à partir des dossiers de laboratoire des patients. Les taux de résistance des isolats d'A. baumannii étaient élevés pour la céftriaxone, la céfotaxime et la ticarcilline (100\%), la ceftazidime, la céfépime et la pipéracilline (98,3\%), l'imipénème (97,4\%), la pipéracilline/le tazobactam (96,6\%), les quinolones (94,8 \%), I'ampicilline/le sulbactam (89,7 \%), la gentamicine (87,9\%), le tobramycine et la tétracycline (76,7 \%) et le triméthoprime/le sulfaméthoxazole (75,9\%), mais étaient moins élevés pour la minocycline (26,7 \%) et la colistine (1,7 \%). Dans notre hôpital, $A$. baumannii était très résistant à tous les antibiotiques, notamment à la tigécycline, sauf à la minocycline et à la colistine, qui étaient considérées comme le traitement de dernier recours contre les souches d'A. baumannii multirésistantes.

'Intensive Care Department, Al-Hussein Hospital, King Hussein Medical Centre, Amman, Jordan (Correspondence to A. Batarseh: adelbatarseh@ gmail.com). ${ }^{2}$ Department of Microbiology, Princess Iman Centre for Research and Laboratory Science, King Hussein Medical Centre, Amman, Jordan. ${ }^{3}$ Department of Pharmacy, Al-Hussein Hospital, King Hussein Medical Centre, Amman, Jordan.

Received: 02/12/14; accepted: 29/07/15 


\section{Introduction}

Acinetobacter baumannii resistant to multiple antimicrobial agents has been increasing worldwide over recent decades. This resistance pattern usually varies with time, and from one region to another or even within the same region (1). Furthermore, A. baumannii has become one of the most problematic multidrug resistant (MDR) pathogens in the health care environment and is responsible for many nosocomial infection outbreaks, especially in critical care areas. These include bloodstream, soft tissue, urinary tract, central nervous system and surgical site infections and ventilator-associated pneumonia (1-3), all of which have been associated with high mortality rates and treatment costs (4).

There is limited information in Jordan regarding the epidemiology, prevalence and resistance rates of A. baumannii isolates from different sites of infection (5-7). This study was therefore conducted to determine the current prevalence and the local antibiogram of MDR-AB isolates from different sites of infection in a tertiary teaching hospital at King Hussein Medical Centre, Amman.

\section{Methods}

This was a retrospective study conducted in the department of microbiology, Princess Iman Centre for Research and Laboratory Sciences, King Hussein Medical Centre, Amman, over a period of 12 months from January to December 2013. The study protocol was approved by the ethics committee of the Royal Medical Services.

\section{Sampling}

From a total of 374 positive cultures of A. baumannii 116 non-repetitive positive clinical samples for A. baumannii from various sources, including blood $(n=24)$, sputum $(n=28)$, urine $(n=$ $7)$ and pus swabs $(n=57$ from wounds, tips of catheters and body parts) were retrieved from patients' laboratory records at Al-Hussein Hospital, King Hussein Medical Centre.

\section{Data collection}

Clinical and Laboratory Standards Institute (CLSI) recommendations for 2012 were adopted for culture, isolation and identification of all A. baumannii isolates and for antibiotic susceptibility testing using the VITEK 2 Compact automated microbiology system (bioMérieux), with 2 complementary sets of antibiotic susceptibility testing (AST) cards (AST-N233 and AST-XN05) (8). Samples that were tested manually or against only one of the AST-cards or to different AST-cards were excluded, i.e. only samples that were tested against both AST cards were included in the study. The minimal inhibitory concentration (MIC) interpretive standards for $A$. baumannii were adopted from the CLSI guideline 2012 (8) for the following groups of antibiotics:

- group I: penicillins (ticarcillin and piperacillin), beta-lactames/betalactamase-inhibitor combinations (ampicillin/sulbactam and piperacillin/tazobactam), 3rd and 4th generation cephalosporins (ceftazidime, cefotaxime ceftriaxone, and cefepime);

- group II: carbapenems (imipenem);

- group III: fluoroquinolones (ciprofloxacin and levofloxacin);

- group IV: aminoglycosides (tobramycin and gentamicin);

- group V: tetracyclines (minocycline and tetracycline);

- group VI: folate pathway inhibitors (sulfamethoxazole/trimethoprim);

- group VII: lipopeptides (colistin), and potential antimicrobial agents; and

\section{- tigecycline.}

\section{Definitions}

Since there is no agreed single definition for MDR and pan-drug resistance
(PDR) for A. baumannii in the literature $(9,10)$ the following definitions were adopted in this study. MDR was defined as resistance to imipenem plus 3 or more different antibiotic classes, including: at least 2 beta-lactames (penicillin, betalactames/beta-lactamase-inhibitor combinations, 3rd- and 4th-generation cephalosporins); tobramycin or gentamicin; ciprofloxacin or levofloxacin; tetracyclines; or sulfamethoxazole/ trimethoprim. PDR was defined as resistance to all tested antibiotics or only susceptible to colistin. Tigecycline was not included in this definition since no agreed breakpoints for tigecycline have been approved by the CLSI 2012 guideline (8).

\section{Results}

Over a period of 12 months, a total of 116 A. baumannii isolates were found. The distribution according to their site of infection is shown in Table 1. Isolates were obtained from swabs (49.1\%), blood (24.2\%), sputum (20.7\%) and urine (6.0\%). More of the isolates were from male $(73,62.9 \%)$ than female patients.

A. baumannii resistance to various antibiotics groups is summarized in $\mathrm{Ta}$ ble 2. The percentage of resistant A. baumannii from various sources was highest for ceftriaxone, cefotaxime, and ticarcillin $(100 \%)$, followed by ceftazidime, cefepime, and piperacillin (98.3\%), while resistance to other tested antibiotics were: imipenem (97.4\%), piperacillin/ tazobactam (96.6\%), ciprofloxacin and levofloxacin (94.8\%), ampicillin/sulbactam (89.7\%), gentamicin (87.9\%), tobramycin and tetracycline (76.7\%), sulfamethoxazole/trimethoprim (75.9\%). On the other hand, the rate of resistance of $A$. baumannii isolates was remarkably low for colistin (1.7\%) and minocycline (26.7\%).

The resistance pattern differed significantly across samples of different origins $(P \leq 0.05)$ for quinolones, 


\begin{tabular}{|c|c|c|c|c|c|c|}
\hline \multirow[t]{2}{*}{ Specimen } & \multicolumn{2}{|c|}{ Males } & \multicolumn{2}{|c|}{ Females } & \multicolumn{2}{|c|}{ Total } \\
\hline & No. & $\%$ & No. & $\%$ & No. & $\%$ \\
\hline Blood & 17 & 14.7 & 7 & 6.0 & 24 & 20.7 \\
\hline Sputum & 16 & 13.8 & 12 & 10.3 & 28 & 24.2 \\
\hline Swab & 33 & 28.4 & 24 & 20.7 & 57 & 49.1 \\
\hline Urine & 7 & 6.0 & 0 & 0.0 & 7 & 6.0 \\
\hline Total & 73 & 62.9 & 43 & 37.1 & 116 & 100.0 \\
\hline
\end{tabular}

tetracyclines, gentamicin and sulfamethoxazole/trimethoprim. In addition, antibiotic resistance was lowest with urine samples for sulfamethoxazole/ trimethoprim (42.9\%) and with sputum samples for ampicillin/sulbactam (78.6\%), followed by blood samples, especially with ciprofloxacin and levofloxacin $(75.0 \%)$, gentamicin $(70.8 \%)$, tobramycin $(66.7 \%)$, tetracycline (58.3\%) and minocycline (12.5\%). Since there are no agreed breakpoints for tigecycline against $A$. baumannii in the CLSI guidelines or in the literature $(8,11)$, we used the AST-XN05 card MIC breakpoints of susceptibility (susceptible $\leq 0.5 \mathrm{mg} / \mathrm{L}$, resistant $\geq 8$ $\mathrm{mg} / \mathrm{L})$. On this basis, 20 (17.2\%) isolates were reported to be susceptible to tigecycline (Table 3).

According to our definitions, 90 A. baumannii isolates were multidrug resistant $(77.6 \%)$, while 10 isolates were pan-drug resistant (8.6\%) (Table 4).

\section{Discussion}

In the last decades, $A$. baumannii has been considered as one of the most resistant bacteria within the hospital environment, especially in critical care areas, which are responsible for the most severe nosocomial infections. These infections often start locally, then progress to bacteraemia and even septicaemia $(1,2,12,13)$ due to several contributing factors, for example, inappropriate initial antimicrobial therapy, early interruption of treatment, sub-therapeutic doses, minimal tissue penetration and
MDR, and also as a result of the overuse of 3rd-generation cephalosporin (13), quinolones or broad-spectrum antibiotics (14), and to a lack of proper instrument decontamination and personal hygiene (2,13). For these reasons, the A. baumannii resistance patterns differ internationally, regionally and locally in developing and developed countries (1).

More of the A. baumannii isolates identified in our study were from male $(62.9 \%)$ than female patients, in agreement with observations in previous studies $(11-12)$. The most common source of $A$. baumannii isolates was swabs (49.1\%), followed by blood (24.2\%), sputum (20.7\%) and urine (6.0\%). The A. baumannii resistance pattern across various sample origins was significantly different for quinolones, tetracyclines, gentamicin and sulfamethoxazole/trimethoprim. The different resistance pattern for A. baumannii from different sample sources is in agreement with previous findings from King Hussein Medical Centre in a 2001 study, and further investigations are needed to elucidate the cause of these differences (6).

In the present study, isolates of $A$. baumannii showed a high resistance rate (94-100\%) to all generations of cephalosporins, penicillins, imipenem and quinolones, findings which are in general similar to the results of studies in Jordan, Islamic Republic of Iran, India and Italy, and a little higher than those from studies in Malaysia, Turkey and the United States of America (USA) $(50-78 \%)(7,15,16)$.
The high resistance rates of isolates to penicillins and beta-lactamase inhibitors in the present study (89-96\%) were consistent with the results from other studies obtained from Jordan, Islamic Republic of Iran, India, Turkey and Italy for piperacillin/tazobactam (5,15-19), but were higher than the results from south India (39\%) and Malaysia (72\%) (20,21). At the same time, lower resistance rates for ampicillin/sulbactam were found in studies in Italy and Malaysia (47.5\% and 68.5\% respectively) $(18,19)$.

Historically, carbapenems have been considered the best therapeutic option for infections caused by MDR A. baumannii. Recently, carbapenemresistant $A$. baumannii have been increasing worldwide, reaching an alarmingly high level in some countries, such as Turkey (78\%), Islamic Republic of Iran (86\%) and India (89.6\%). At the same time, A. baumannii in Jordan demonstrated high resistance rates to meropenem (73.4-100\%), while imipenem showed lower resistance rates (63-73.2\%) in general, but this resistance usually varies over time, even at King Hussein Medical Centre, where it was only $6.7 \%$ in $2001(5-7,15,16,18)$. Nevertheless, carbapenems are still considered one of the treatment options for MDR A. baumannii, which retains sensitivity to carbapenems. However, $A$. baumannii resistance to imipenem is still low in some studies, even from the same countries that were associated with high resistance rates: Islamic Republic of $\operatorname{Iran}(26.5 \%)$ and India (4.5\%) (20,22). For carbapenem-resistant A. baumannii, 


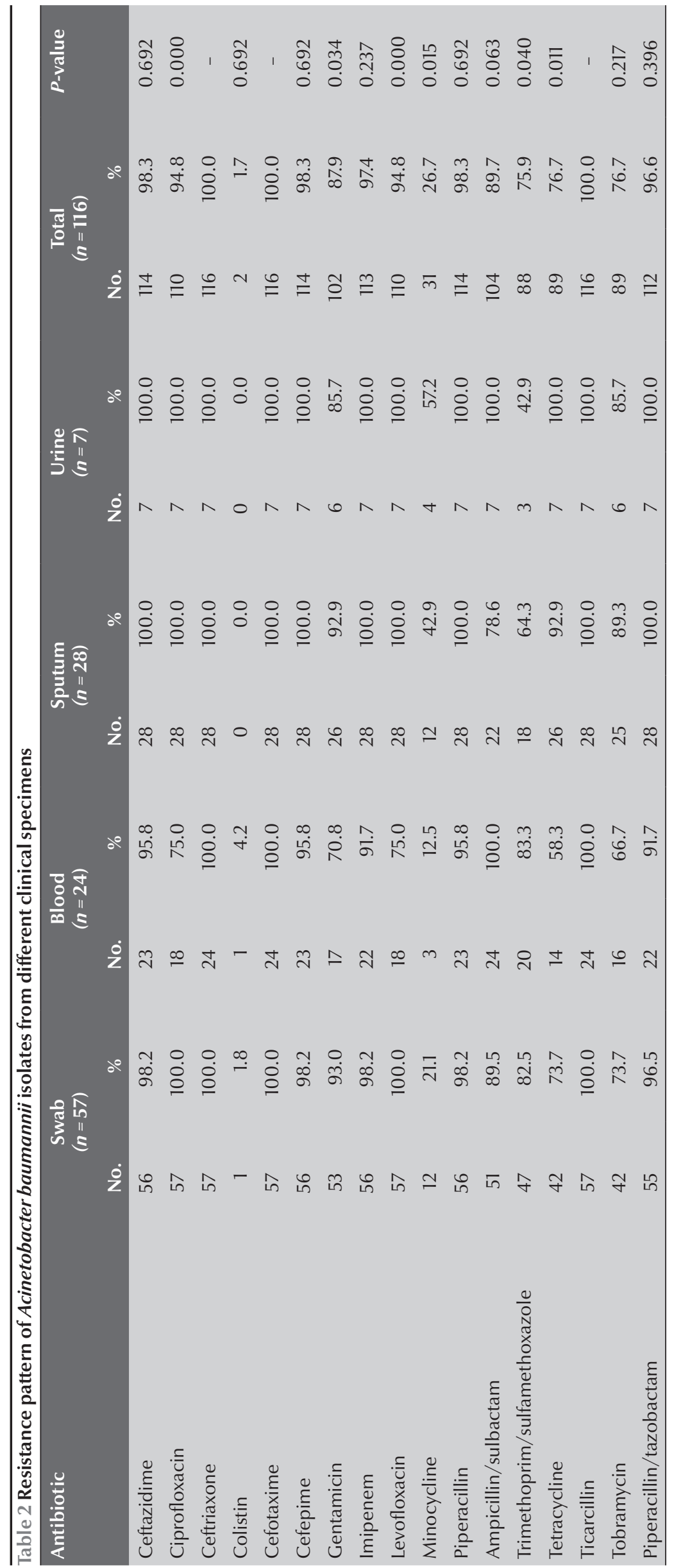

tigecycline and colistin are 2 of the most frequently used alternative agents according to the literature (23).

A. baumannii isolates in our hospital showed high resistance rates to aminoglycosides $(87.9 \%$ and $76.7 \%$ for gentamicin and tobramycin respectively). The gentamicin resistance rate was also in line with the results from Islamic Republic of Iran and India $(15,17,18)$. At the same time, the resistance rate was found to be lower (around 60\%) in other countries such as south India, Malaysia and Italy. On the other hand, resistance rate of $37.9 \%$ and $70.8 \%$ for tobramycin and gentamicin respectively were recorded at King Hussein Medical Centre in 2001 (6,7,19-21). However, tobramycin resistance from Italy (44.3\%) was much lower than in this study and in studies from Malaysia $(64.8 \%)$ and India $(80 \%)(18,19,21)$.

A. baumannii isolates have been found to have a variable degree of resistance to the sulfamethoxazole/ trimethoprim combination, ranging from $59.8 \%$ in south India, $80 \%$ in Italy and up to $100 \%$ in Islamic Republic of Iran $(15,19,20)$. In comparison with these previous reports, $75.9 \%$ of the A. baumannii isolates from the present study were found to be resistant to sulfamethoxazole/trimethoprim, while only $65.6 \%$ of the isolates were resistant at our Centre in 2001 (6).

The tetracycline resistance rate in this study was $76.7 \%$, which is lower than rates from Malaysia (87\%), Italy (93.8\%) and Islamic Republic of Iran $(100 \%)(15,19,21)$. On the other hand, the minocycline resistance rate was low in the present study (26.7\%), which is similar to what was reported from Italy $(21.3 \%)$, indicating that minocycline is one of the best antibiotics that can be used in combination with the other anti-Acinetobacter antibiotics (19).

Tigecycline is a parenteral broadspectrum bacteriostatic minocycline derivative. It has been used alone or in combination with other 


$\begin{aligned} & \text { Table } 3 \text { Minimal inhibitory concentration }(\mathrm{MIC}) \text { values for tigecycline in the tested } \\
& \text { Acinetobacter baumannii isolates }(\boldsymbol{n}=\mathbf{1 1 6})\end{aligned}$
\begin{tabular}{lcc}
\hline Tigecycline MIC $(\mathbf{m g} / \mathrm{L})$ & No. of isolates & $\%$ \\
$\leq 0.5$ & 20 & 17.2 \\
1.0 & 27 & 23.3 \\
2.0 & 49 & 42.2 \\
4.0 & 15 & 12.9 \\
$\geq 8.0$ & 5 & 4.3 \\
\hline
\end{tabular}

anti-Acinetobacter drugs for MDR A. baumannii, but with varying degrees of success $(15,18,19,23,24)$. According to the European Committee on Antimicrobial Susceptibility Testing (EUCAST), the United States Food and Drug Administration (FDA) and the CLSI, there are no specific breakpoints for tigecycline as an anti-Acinetobacter agent $(21,24,25)$. On the other hand, the British Society for Antimicrobial Chemotherapy (BSAC) has previously recommended the $\leq 1 \mathrm{mg} / \mathrm{L}$ breakpoint of susceptibility, but recently applied the EUCAST recommendations of "non-speciesspecific MIC breakpoint of susceptibility $=0.25 \mathrm{mg} / \mathrm{L}$ and $R=>0.5 \mathrm{mg} / \mathrm{L}$ to interpret susceptibility, while other studies recommended $\leq 2 \mathrm{mg} /$ L breakpoints" (25-27). However, we considered the AST-XN05 card MIC of $\leq 0.5$ $\mathrm{mg} / \mathrm{L}$ to be the MIC breakpoint of susceptibility, as shown in Table 3. A study from Jordan showed no resistance to tigecycline (0\%), even at MIC 1.5-2.0 $\mathrm{mg} / \mathrm{L}(7)$. At the same time, tigecycline resistance in the present study was $17.3 \%, 59.5 \%$ and $82.8 \%$ according to the MIC breakpoints of $\leq 2.0 \mathrm{mg} / \mathrm{L}, \leq$ $1.0 \mathrm{mg} / \mathrm{L}$ and $\leq 0.5 \mathrm{mg} / \mathrm{L}$ respectively. Due to the wide range of resistance to tigecycline, there is a growing need for agreed breakpoints of susceptibility to be declared and accepted by CLSI, EUCAST, BSAC, FDA and other institutions. Tigecycline resistance rates were low in studies from Jordan (0\%) and Italy (27.5\%), moderately high in India (74.8\%) and high in this study (82.8\%) and one from Islamic Republic of Iran (98\%) $(7,5,18,19)$.

Colistin is still considered to be the most effective single antibiotic against MDR A. baumannii, and is always kept as a last resort (23) due to the growing rates of resistance to carbapenems in recent decades $(15,16,19)$. At the same time, resistance and treatment failure rates have been increasing with colistin in some countries lately, and therefore different combinations of colistin with other anti-A baumannii antibiotics have been tried, with varying success rates $(23,24)$. In the present study the resistance rate of isolates to colistin was very low $(1.7 \%)$, which is consistent with data reported from Jordan (0\%), India (1.2\%), Italy (1.2\%) and Islamic Republic of $\operatorname{Iran}(7 \%)(7,15,18,19)$. However, resistance rates to colistin were as high as $25.9 \%, 30.6 \%$ and $40.7 \%$ in Malaysia, Spain and Korea respectively, presumably due to the intensive use of colistin recently $(7,15,21,23,28,29)$.

\begin{tabular}{|c|c|c|}
\hline Variable & No. of isolates & Susceptibility (\%) \\
\hline Multidrug resistant ${ }^{\mathrm{a}}$ & 90 & 77.6 \\
\hline Colistin + minocycline & 37 & 31.9 \\
\hline Colistin + tobramycin & 1 & 0.9 \\
\hline Colistin + sulfamethoxazole/trimethoprim & 12 & 10.3 \\
\hline Colistin + ampicillin/sulbactam & 6 & 5.2 \\
\hline Colistin + ampicillin/sulbactam + sulfamethoxazole/trimethoprim & 2 & 1.7 \\
\hline Colistin + sulfamethoxazole/trimethoprim + tobramycin & 1 & 0.9 \\
\hline Colistin + minocycline + ampicillin/sulbactam & 1 & 0.9 \\
\hline Colistin + minocycline + sulfamethoxazole/trimethoprim & 9 & 7.8 \\
\hline Colistin + minocycline + tetracycline & 10 & 8.6 \\
\hline Colistin + minocycline + tobramycin & 2 & 1.7 \\
\hline Colistin + minocycline + tetracycline + tobramycin + sulfamethoxazole/trimethoprim & 9 & 7.8 \\
\hline Pandrug resistant ${ }^{b}$ & 10 & 8.6 \\
\hline Not susceptible to any of the tested antibiotics & 2 & 1.7 \\
\hline Susceptible only to colistin & 8 & 6.9 \\
\hline
\end{tabular}

${ }^{a}$ Resistant to imipenem plus 3 or more different antibiotic classes.; ${ }^{b}$ Resistant to all tested antibiotics or only susceptible to colistin; ${ }^{c}$ Percentage of total number of isolates tested $(n=116)$ susceptible to colistin only. 
The extensive use of such broadspectrum antibiotics as 3rd-generation cephalosporins, quinolones and carbapenems has been associated with emergence of MDR A. baumannii (13-15,18). Moreover, PDR to all available antibiotics has been reported worldwide, even against colistin, minocycline, tigecycline and sulbactam, which dictates the use of combinations of antibiotics, albeit with variable success rates $(30,31)$. The current study reported high MDR resistance rates of A. baumannii against most antibiotics tested $(77.6 \%)$. The highest rates have been reported from Israel (88\%), while lower resistance rates (around $72 \%$ ) were found in Malaysia, USA and south India $(20,21,32)$. Finally, the most effective antibiotics against A. baumannii according to the current study were colistin (98.3\%) and minocycline (73.3\%), followed by sulfamethoxazole/trimethoprim (24.1\%), then tetracycline and tobramycin (23.3\%). Interestingly, tigecycline showed a high resistance rate $(82.8 \%)$ when we considered the VITEK 2 system ASTXN05 card breakpoints $(\leq 0.5 \mathrm{mg} / \mathrm{L})$. However, only $8.6 \%$ of our A. baumannii isolates were reported to be PDR, which is lower than the south India results
(17.2\%) (20). In addition, the colistin and minocycline combination seems to be the most effective combination theoretically $(31.9 \%)$, with a good bactericidal activity, as shown in Table 4 . The prescription of colistin and minocycline should therefore be guided by the antibiotic protocols and only be ordered by infectious disease specialists in order to minimize the risk of side-effects and rising resistance rates and treatment failure rates (33).

The present study had some limitations due to the retrospective study design. Patients' data were missing or incomplete in many cases for data such as age, comorbidity and patient's location. Future studies with larger sample sizes are necessary to take into account other contributing factors such as irrational use of antibiotics, lack of strict application of infection control instructions, isolation measures, patient and staff hygiene and environmental decontamination. Studies are also needed into the prevalence of MDR and PDR in acute care settings, how to distinguish between colonization (which does not require antibiotic treatment) and infections (which might require antibiotics), and to assess the associated costs of treatment and the associated mortality rates.

\section{Conclusions}

A. baumannii isolates in Al-Hussein Hospital of Hussein Medical Centre in Amman were found to be highly resistant to almost all tested antibiotics, up to an alarming level, except mainly for colistin and minocycline, which showed relatively low resistance rates. However, the effectiveness and safety of colistin and minocycline need to be thoroughly investigated in the future. Therefore, it is important to create a new well-designed protocols or guidelines for both antibiotic use and isolation measures to help minimize the development and the spread of these MDR and PDR A. baumannii isolates in different hospital wards, crossinfection between patients, morbidity and mortality rates and, finally, the cost of treatment. Moreover, protocol-specified reviews of antibiotic susceptibility of all A. baumannii isolates is mandatory for escalation or de-escalation of antibiotic use.

\section{Acknowledgements}

\section{Funding: None.}

Competing interests: None declared.

\section{References}

1. Maragakis LL, Perl TM. Acinetobacter baumannii: epidemiology, antimicrobial resistance, and treatment options. Clin Infect Dis. 2008 Apr 15;46(8):1254-63. PMID:18444865

2. Fournier PE, Richet $\mathrm{H}$. The epidemiology and control of Acinetobacter baumannii in health care facilities. Clin Infect Dis. 2006 Mar 1;42(5):692-9. PMID:16447117

3. Peleg AY, Seifert H, Paterson DL. Acinetobacter baumannii: emergence of a successful pathogen. Clin Microbiol Rev. 2008 Jul;21(3):538-82. PMID:18625687

4. Brusselaers N, Vogelaers D, Blot S. The rising problem of antimicrobial resistance in the intensive care unit. Ann Intensive Care. 2011;1:47. 10.1186/2110-5820-1-47 PMID:22112929

5. Dhabaan GN, Hamimah H, Shorman MA. Emergence of extensive drug-resistant Acinetobacter baumannii in North of Jordan. Afr J Microbiol Res. 2011;5(9):1070-4.

6. Abu Setteh MH. Antibiotic resistance patterns of Acinetobacter species isolated in King Hussein Medical Center, Jordan. Saudi Med J. 2005 Jan;26(1):153-5. PMID:15756380

7. Al-Dabaibah N, Obeidat NM, Shehabi AA. Epidemiology features of Acinetobacter baumannii colonizing respiratory tracts of ICU patients. International Arabic Journal of Antimicrobial Agents. 2012;2(2).

8. Performance standards for antimicrobial susceptibility testing. 22nd informational supplement, M100-S22. Wayne (PA): Clinical and Laboratory Standards Institute; 2012.

9. Falagas ME, Koletsi PK, Bliziotis IA. The diversity of definitions of multidrug-resistant (MDR) and pandrug-resistant (PDR) Acinetobacter baumannii and Pseudomonas aeruginosa. J Med Microbiol. 2006 Dec;55(Pt 12):1619-29. PMID:17108263

10. Falagas ME, Karageorgopoulos DE. Pandrug resistance (PDR), extensive drug resistance (XDR), and multidrug resistance (MDR) among Gram-negative bacilli: need for international harmonization in terminology. Clin Infect Dis. 2008 Apr 1;46(7):1121-2, author reply 1122. PMID:18444833

11. Brink AJ, Bizos D, Boffard KD, Feldman C, Grolman DC, Pretorius J, et al.; Association of Surgeons of South Africa; Critical Care Society of Southern Africa; Federation of Infectious Diseases Societies of Southern Africa; South African Thoracic Society; Trauma Society of South Africa. Guideline: appropriate use of tigecycline. S Afr Med J. 2010 Jun;100(6 Pt 2):388-94. PMID:20529440 
12. Wilson SJ, Knipe CJ, Zieger MJ, Gabehart KM, Goodman JE, Volk HM, et al. Direct costs of multidrug-resistant Acinetobacter baumannii in the burn unit of a public teaching hospital. Am J Infect Control. 2004 Oct;32(6):342-4. PMID:15454892

13. Fierobe L, Lucet JC, Decré D, Muller-Serieys C, Deleuze A, Joly-Guillou ML, et al. An outbreak of imipenem-resistant Acinetobacter baumannii in critically ill surgical patients. Infect Control Hosp Epidemiol. 2001 Jan;22(1):35-40. PMID:11198020

14. Bayuga S, Zeana C, Sahni J, Della-Latta P, el-Sadr W, Larson E. Prevalence and antimicrobial patterns of Acinetobacter baumannii on hands and nares of hospital personnel and patients: the iceberg phenomenon again. Heart Lung. 2002 Sep-Oct;31(5):382-90. PMID:12487017

15. Japoni-Nejad A, Sofian M, van Belkum A, Ghaznavi-Rad E. Nosocomial outbreak of extensively and pandrug-resistant Acinetobacter baumannii in tertiary hospital in central part of Iran. Jundishapur JMicrobiol. 2013;6(8):1-5.

16. Bacakoğlu F, Korkmaz Ekren P, Taşbakan MS, Başarik B, Pullukçu H, Aydemir S, et al. Solunumsal yogun bakim unitesinde coklu antibiyotik direncli Acinetobacter baumanni enfeksiyonu. [Multidrug-resistant Acinetobacter baumannii infection in respiratory intensive care unit]. Mikrobiyol Bul. 2009 Oct;43(4):575-85. PMID:20084910

17. Davood Y, Shahram A, Latif G, et al. Prevalence and drug resisf tance of Acinetobacter baumannii in ICU of a teaching hospital. J. Appl. Environ. Biol. Sci. 2013;3(9):22-7.

18. Jaggi N, Sissodia P, Sharma L. Acinetobacter baumannii isolates in a tertiary care hospital: antimicrobial resistance and clinical significance. J Microbiol Infect Dis. 2012;2(2):57-63.

19. Alessandro C, Silvia D, Paolo V, et al. In vitro activity of tigea cycline against multidrug resistant Acinetobacter baumannii]. Antimicrob Chemother. 2008;62(2):422-42.

20. Sivaranjani V, Umadevi S, Srirangaraj S, Kali A, Seetha K. Multidrug resistant Acinetobacter species from various clinical samples in a tertiary care hospital from South India. Australas Med J. 2013;6(12):697-700. PMID:24391681

21. Soo-Sum L, Zarizal S, Salwani I, Rahman NIA, Othman N ,Abdullah $\mathrm{FH}$, et al. Prevalence and genetic characterization of polymyxin-resistant Acinetobacter baumannii isolated from a tertiary hospital in Terengganu, Malaysia. ISRN Microbiol. 2014 Mar 19;2014:953417 PMID:25006521

22. Rahbar M, Mehrgan H, Aliakbari NH. Prevalence of antibioticresistant Acinetobacter baumannii in a 1000-bed tertiary care hospital in Tehran, Iran. Indian J Pathol Microbiol. 2010 AprJun;53(2):290-3. PMID:20551535

23. Cai Y, Chai D, Wang R, Liang B, Bai N. Colistin resistance of Acinetobacter baumannii: clinical reports, mechanisms and antimicrobial strategies. J Antimicrob Chemother. 2012 Jul;67(7):1607-15. PMID:22441575

24. Falagas ME, Kasiakou SK. Colistin: the revival of polymyxins for the management of multidrug-resistant Gram-negative bacterial infections. Clin Infect Dis. 2005 May 1;40(9):1333-41. PMID:15825037

25. Casal M, Rodríguez F, Johnson B, Garduno E, Tubau F, de Lejarazu RO, et al. Influence of testing methodology on the tigecycline activity profile against presumably tigecycline-nonsusceptible Acinetobacter spp. J Antimicrob Chemother. 2009 Jul;64(1):69-72. PMID:19451133

26. BSAC methods for antimicrobial susceptibility testing, version 12 May 2013 [Internet]. Birmingham: British Society for Antimicrobial Chemotherapy; 2013 (http://www.bsac.org. uk/wp-content/uploads/2012/02/Version-12-Apr-2013_final. pdf, accessed 13 August 2105).

27. Karageorgopoulos DE, Kelesidis T, Kelesidis I, Falagas ME. Tigecycline for the treatment of multidrug-resistant (including carbapenem-resistant) Acinetobacter infections: a review of the scientific evidence. J Antimicrob Chemother. 2008 Jul;62(1):45-55. PMID:18436554

28. Arroyo LA, Mateos I, González V, Aznar J. In vitro activities of tigecycline, minocycline, and colistin-tigecycline combination against multi- and pandrug-resistant clinical isolates of Acinetobacter baumannii group. Antimicrob Agents Chemother. 2009 Mar;53(3):1295-6. PMID:19075049

29. Ko KS, Suh JY, Kwon KT, Jung SI, Park KH, Kang Cl, et al. High rates of resistance to colistin and polymyxin B in subgroups of Acinetobacter baumannii isolates from Korea. I Antimicrob Chemother. 2007 Nov;60(5):1163-7. PMID:17761499

30. Falagas ME, Bliziotis IA, Kasiakou SK, Samonis G, Athanassopoulou P, Michalopoulos A. Outcome of infections due to pandrug-resistant (PDR) Gram-negative bacteria. BMC Infect Dis. 2005;5:24. PMID:15819983

31. Valencia R, Arroyo LA, Conde M, Aldana JM, Torres MJ, Fernández-Cuenca $\mathrm{F}$, et al. Nosocomial outbreak of infection with pan-drug-resistant Acinetobacter baumannii in a tertiary care university hospital. Infect Control Hosp Epidemiol. 2009 Mar;30(3):257-63. PMID:19199531

32. Abbo A, Navon-Venezia S, Hammer-Muntz O, Krichali T, Siegman-Igra Y, Carmeli Y. Multidrug-resistant Acinetobacter baumannii. Emerg Infect Dis. 2005 Jan;11(1):22-9. PMID:15705318

33. Tan TY, Ng LS, Tan E, Huang G. In vitro effect of minocycline and colistin combinations on imipenem-resistant Acinetobacter baumannii clinical isolates. J Antimicrob Chemother. 2007 Aug;60(2):421-3. PMID:17540671 